\title{
Bilateral Medial Medullary Infarction Accompanied by Cerebral Watershed Infarction: A case report
}

\author{
Jingmin Zhao ${ }^{1,2}$, Guangxian Nan $^{1 *}$, Guangxun Shen ${ }^{1}$, Songji Zhao ${ }^{3,4}$, Hiroshi Ito ${ }^{2,3}$ \\ 1. Department of Neurology, China-Japan Union Hospital of Jilin University, Changchun, China \\ 2. Department of Radiology and Nuclear Medicine, Fukushima Medical University, Fukushima, Japan \\ 3. Advanced Clinical Research Center, Fukushima Global Medical Science Center, Fukushima Medical University, Fukushima, Japan \\ 4. Basic Medical College of Jilin University, Changchun, China
* Correspondence: Guangxian Nan, MD, PhD, Department of Neurology, China-Japan Union Hospital of Jilin University, 126 XianTai Street, Changchun, 130031, Jilin, China

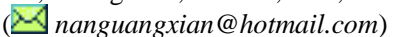

Radiology Case. 2020 Apr; 14(4):1-7 $\quad$ :: $\quad$ DOI: $10.3941 /$ jrcr.v14i4.3905

\begin{abstract}
Bilateral medial medullary infarction is a rare stroke subtype, and its diagnosis has become possible by brain magnetic resonance imaging. In this report, we describe a case in which acute bilateral medial medullary infarction accompanied by cerebral watershed infarction was clearly identified by diffusion-weighted imaging, and we discuss the mechanisms of bilateral medial medullary infarction accompanied by cerebral watershed infarction.
\end{abstract}

\section{CASE REPORT}

\section{CASE REPORT}

A 50-year-old man was admitted to our hospital with sensory dysfunction and pain in the four limbs, which began 2 hours before admission. The patient's condition, however, worsened into a rapidly progressive tetraparesis with slurred speech and dysphagia within 3 hours after admission. He had a history of hypertension, cigarette smoking, and myocardial infarction. Neurologic examination revealed breathing difficulty, dysarthria, hyporeflexia, quadriplegia, diminished pain sensation, and a muscle power of grade 4 for his four limbs. His National Institutes of Health Stroke Scale score was 12/42. Brain magnetic resonance imaging (MRI) performed 11 hours after admission showed the 'heart appearance' sign detected as a hyperintense signal in the bilateral anteromedial medullae (Fig. 1A), cortical watershed infarction (CWI) (Fig. 1B), and internal watershed infarction (IWI) (Fig. 1C) of the right cerebral hemisphere shown by diffusion-weighted imaging (DWI), respectively. He then underwent a brain digital subtraction angiography (DSA), which showed mild stenosis in the basilar artery (BA) (Fig. 2A), plaque formation in the right internal carotid artery (ICA) without obvious stenosis (Fig. 2B), mild stenosis in the right middle cerebral artery (MCA) (Fig. 2C), and a normal right vertebral artery (VA) (Fig. 2D), which appeared more dominant than the left in the extracranial portion (Fig. 2E). The left VA was occluded at the distal intracranial portion (Fig. 2F). Two days after admission, the patient required an endotracheal intubation because of respiratory distress with disorder of consciousness (Glasgow Coma Scale score, 3/15) and a tracheotomy mask on day 3 of hospitalization. On day 10 of hospitalization, his consciousness improved (Glasgow Coma Scale score, 2+2+T/15) and his respiratory distress significantly improved; however, his four limbs remained weak. The patient had a modified Rankin Scale score of 5/6 at the time of his discharge without further treatment. 


\section{DISCUSSION}

\section{Etiology \& Demographics:}

Medial medullary infarction (MMI) is a rare stroke subtype, accounting for only $0.5-1.5 \%$ of all strokes [1,2], and bilateral MMI is even rarer [3]. Clinicopathologic studies have demonstrated that MMI is a rare condition, and in a series of 700 patients who died of cerebrovascular disease and were autopsied, only four were found to have ischemic damage in the medial medullae [4]. Bilateral MMI is even more rarely reported, and it is speculated that it may be related to an unpaired anterior spinal artery as an anatomical variation $[5,6]$. The etiological classifications of infarctions are largeartery atherosclerosis (LA), small-vessel disease (SV), arterial dissection (DI), cardiac embolism (CE), and stroke of undetermined etiology. Risk factors for cerebral infarction include atrial fibrillation, hypertension, smoking habit, diabetes mellitus, ischemic heart disease, and dyslipidemia. According to previous magnetic resonance angiography findings, bilateral MMI might be related to artery stenosis or occlusion, including VA atherosclerosis (38.5\%), VA occlusion (15.4\%), basilar artery atherosclerosis (19.2\%), dissection (7.7\%), anterior spinal artery (ASA) occlusion (3.8\%; an autopsy case), and no abnormalities (38.5\%) [3]. In patients with normal vascular imaging findings, the stroke mechanism was likely atheromatous branch occlusion or ASA occlusion, which could not be demonstrated by DSA [3].

\section{Clinical \& Imaging Findings:}

The diagnosis of bilateral MMI was previously possible only at autopsy; however, its diagnosis has recently become possible by diffusion-weighted brain MRI, which shows the characteristic "heart appearance" sign. Previous case reports of bilateral MMI described the same heart appearance sign on axial MR images [4,5]. It is considered that blood is supplied to these areas by the vertebral and anterior spinal arteries, but it is often difficult to identify the occluded blood vessel because of the vastly complex network formed by these vessels. The heart appearance sign is considered to appear when the infarct occurs in the anteromedial territory and anterolateral territory [7].

\section{Treatment \& Prognosis:}

It is difficult to diagnose bilateral MMI in its early stages. However, when properly diagnosed, its treatment is the same as that of cerebral infarction, including endotracheal intubation when respiratory distress occurs. A systematic review (38 patients, from 1992 to 2011) reported that bilateral MMI was present with quadriplegia in $24 \%$ of patients, dysarthria in 18\%, and hypoglossal palsy in 9\% [3]. Furthermore, approximately 9-24\% of patients with bilateral MMI may develop respiratory failure, which is more prevalent in bilateral MMI than in unilateral MMI [3]. In contrast to unilateral MMI, the clinical outcome of bilateral MMI is usually poor [6]. However, no comparative studies have been reported to date

\section{Differential Diagnoses:}

Bilateral MMI with respiratory failure can be frequently misdiagnosed as Guillain-Barre syndrome (GBS), particularly when the initial symptoms develop into flaccid quadriplegia [8]. Indeed, the patient's medical history is very important. Brain MRI and DWI are also helpful, as they can show the classical heart or $\mathrm{V} / \mathrm{Y}$ appearance at the ventral medulla in patients with bilateral MMI $[7,8]$. Of note, the abnormal MRI or DWI signal may be a small dot or a linear shape at the midline of the medulla in the early stages of bilateral MMI [9]. GBS can also be confirmed on the basis of the cerebrospinal finding of an elevated protein level without pleocytosis at slightly later stages. A key point to differentiate between these syndromes is the evolution of clinical presentation: GBS has a subacute evolution, whereas bilateral MMI has a more acute presentation [8].

\section{Case Discussion:}

To the best of our knowledge, bilateral MMI accompanied by cerebral watershed infarction (WSI) confirmed by DWI has not yet been reported. In this report, we first described a case in which acute bilateral MMI accompanied by WSI was identified by DWI. Below we discuss the causes of MMI and the possible mechanism of WSI.

In the current case, DWI showed bilateral MMI accompanied by WSI. WSI, that is, ischemic lesions between two non-anastomosing main arterial territories, can be classified as either CWI or IWI, which can be further divided into subtypes. CWI is further divided into anterior watershed infarction (AWI, between the anterior cerebral artery and the middle cerebral artery), posterior watershed infarction (PWI, between the middle cerebral artery and the posterior cerebral artery), and mixed-type infarction (AWI plus PWI). IWI is also further divided into partial IWI (P-IWI, a single lesion or chainlike, the so-called "rosary-like" pattern in the centrum semiovale) and confluent IWI (C-IWI, large cigar-shaped infarction alongside the lateral ventricle). The simultaneous occurrence of CWI and IWI is identified as mixed-type infarction [10]. In our case, apart from MMI, the hyperintense "heart appearance" sign in the bilateral anteromedial medullae (Fig. 1A), PWI, in the right cerebral hemisphere (Fig. 1B), and P-IWI in the right cerebral hemisphere (Fig. 1C) were detected by DWI. Most IWIs are accompanied by CWIs. To the best of our knowledge, the simultaneous occurrence of bilateral MMI and WSI has yet to be reported, and the mechanism of WSI is not yet fully understood. Traditionally, HDI has been widely accepted as a cause of WSI [11]. From the clinicians' viewpoint, each case of IWI could be linked to a hemodynamic impairment. Previous studies demonstrated an association between IWI and critical stenosis of ICA. This finding supported the theory that HDI may be the main cause of IWI [10,12]. The relationship between CWI and HDI appears more complicated, with a previous report stating that artery-to-artery embolism might play an important role in isolated CWI [13]. The susceptibility of the internal border-zone area to HDI is probably due to low perfusion pressure in the perforating medullary arteries, the most distal branches of the ICA with insufficient collateral 
supply of deep perforating lenticulostriate arteries [10]. Previous studies $[10,13]$ revealed that the rosary-like infarction in the centrum semiovale, which was identified as P-IWI in those studies, appears to be associated with HDI. However, Moustafa et al. [14] found that in addition to HDI, microemboli might also play a role in the pathogenesis of the rosary-like infarction. If there is no blood flow reduction, an embolic mechanism can be considered. Few studies have thoroughly compared the difference between C-IWI and PIWI. One study showed that critical ICA stenosis was more common in P-IWI patients than in C-IWI patients [10]. In our case, brain MRI showed PWI in the right cerebral hemisphere (Fig. 1B) and P-IWI in the right cerebral hemisphere (Fig. 1C) on DWI images. Furthermore, brain DSA showed mild stenosis in the BA (Fig. 2A), plaque formation in the right ICA without obvious stenosis (Fig. 2B), and mild stenosis in the right MCA (Fig. 2C). Therefore, it is considered that bilateral MMI might be associated with basilar artery atherosclerosis. However, there is no evidence to conclude that bilateral MMI might be associated with HDI or microemboli, which might cause the CWI.

Maybe there is another mechanism; in the current case, brain DSA showed that the right VA was more dominant than the left VA in the extracranial portion, and the left VA was occluded at the distal intracranial portion (Figs. 2A, E, and F). The anatomic variability of the left perforator branches, which supply the bilateral anteromedial arterial or ASAs originating from the left VA, might explain the bilateral MMI with unilateral VA occlusion [15].

\section{TEACHING POINT}

MRI shows the classical heart appearance or V/Y sign at the ventral medulla in patients with bilateral medial medullary infarction. Hemodynamic impairment and microemboli are both the causes of cortical watershed infarction.

\section{REFERENCES}

1. Toyoda K, Imamura T, Saku Y, Oita J, Ibayashi S, Minematsu K, Yamaguchi T, Fujishima M. Medial medullary infarction: analyses of eleven patients. Neurology. 1996;47(5):1141-1147. PMID: 8909419

2. Shono Y, Koga M, Toyoda K, et al. Medial medullary infarction identified by diffusion-weighted magnetic resonance imaging. Cerebrovasc Dis 2010;30:519-524. PMID: 20861624

3. Pongmoragot J, Parthasarathy S, Selchen D, et al. Bilateral medial medullary infarction: a systematic review. J Stroke Cerebrovasc Dis 2013;22:775-780. PMID: 22541608
4. Maeda M, Shimono T, Tsukahara H, Maier SE, Takeda K. Acute bilateral medial medullary infarction: a unique "Heart Appearance" sign by diffusion-weighted imaging. Eur Neurol. 2004;51(4):236-237. PMID: 15159607

5. Thijs RD, Wijman CA, van Dijk GW, van Gijn J. A case of bilateral medial medullary infarction demonstrated by magnetic resonance imaging with diffusion-weighted imaging. J Neurol. 2001;248:339-340. PMID: 11374104

6. Kumral E, Afsar N, Kirbas D, Balkir K, Ozdemirkiran T. Spectrum of medial medullary infarction: clinical and magnetic resonance imaging findings. J Neurol. 2002;249:8593. PMID: 11954873

7. Parsi K, Itgampalli RK, Suryanarayana A, et al. Bilateral medial medullary infarction: heart appearance. Neurol India 2013;61:84-85. PMID: 23466852

8. Ma L, Deng Y, Wang J, et al. Bilateral medial medullary infarction presenting as Guillain-Barre-like syndrome. Clin Neurol Neurosurg 2011;113:589-591. PMID: 21397385

9. Torabi AM. Bilateral medial medullary stroke: a challenge in early diagnosis. Case Rep Neurol Med 2013;2013:274373. PMID: 24198988

10. Li Y, Li M, Zhang X, et al. Clinical features and the degree of cerebrovascular stenosis in different types and subtypes of cerebral watershed infarction. BMC Neurology. 2017; 17:166. PMID: 28851301

11. Hoffman SJ, Yee AH, Slusser JP, et al. Neuroimaging patterns of ischemic stroke after percutaneous coronary intervention. Catheter Cardiovasc Interv. 2015;85(6):10331040. PMID: 25256948

12. Del Sette M, Eliasziw M, Streifler JY, Hachinski VC, Fox AJ, Barnett HJ. Internal borderzone infarction: a marker for severe stenosis in patients with symptomatic internal carotid artery disease. For the North American Symptomatic Carotid Endarterectomy (NASCET) Group. Stroke. 2000;31:631-636. PMID: 10700496

13. Derdeyn CP, Khosla A, Videen TO, et al. Severe hemodynamic impairment and border zone--region infarction. Radiology. 2001;220:195-201. PMID: 11425997

14. Moustafa RR, Momjian-Mayor I, Jones PS, et al. Microembolism versus hemodynamic impairment in rosarylike deep watershed infarcts: a combined positron emission tomography and transcranial Doppler study. Stroke. 2011;42:3138-3143. PMID: 21852602

15. Gillilan LA. The correlation of the blood supply to the human brain stem with clinical brain stem lesions. J Neuropathol Exp Neurol 1964;23:78-108. PMID: 14105311 


\section{FIGURES}

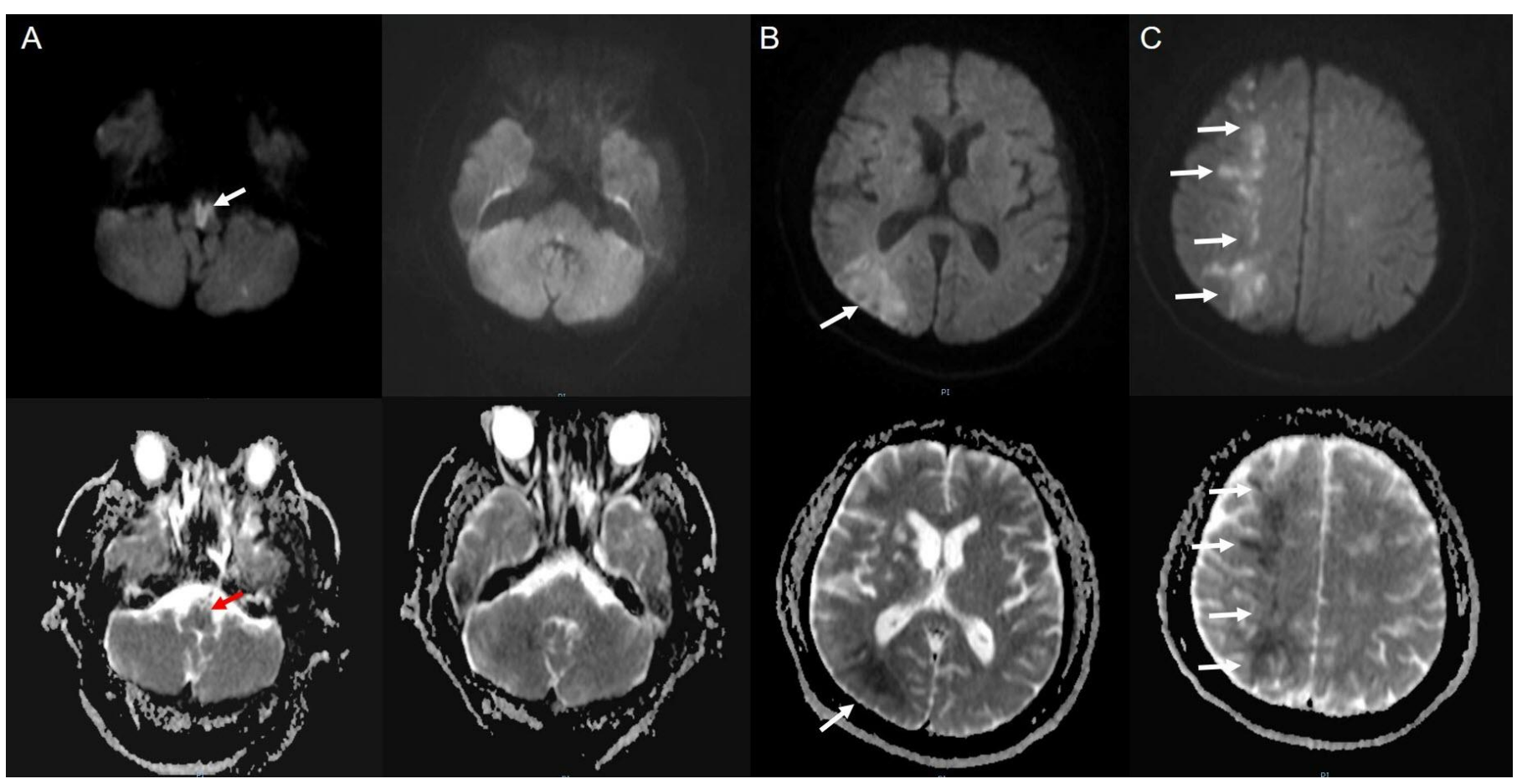

Figure 1: 50-year-old male with medial medullary infarction accompanied by cerebral watershed infarction.

Findings: MRI showed a hyperintense "heart appearance" signal in the bilateral anteromedial medullae on DWI images (top) with corresponding axial apparent diffusion coefficient (ADC) maps (bottom) (Fig. 1A), cortical watershed infarction in the right cerebral hemisphere on DWI images (top) with corresponding ADC maps (bottom) (Fig. 1B) and internal watershed infarction in the right cerebral hemisphere on DWI images (top) with corresponding ADC maps (bottom) (Fig. 1C).

TECHNIQUE: Axial diffusion-weighted 1.5T-MRI of the head. 

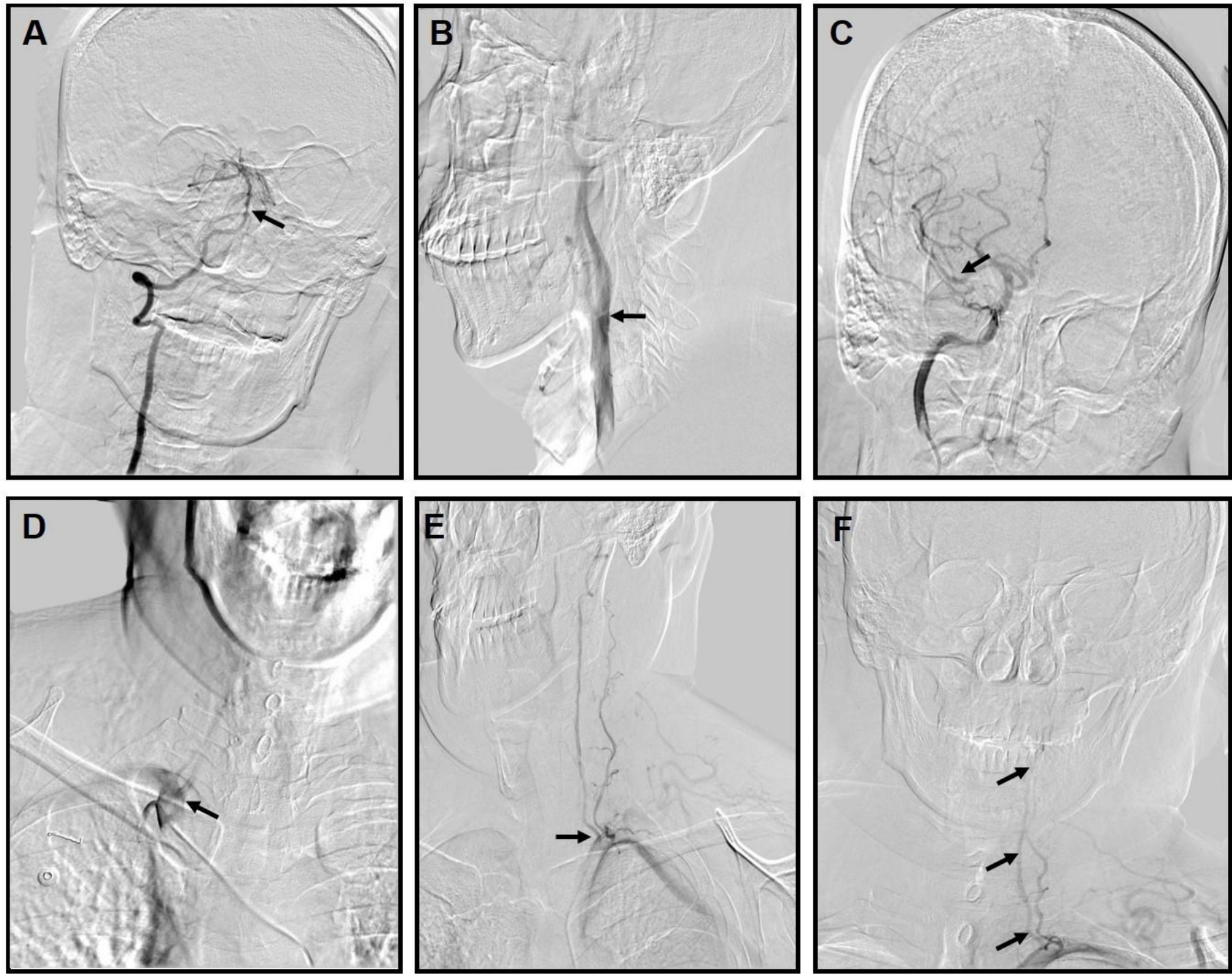

Figure 2: 50-year-old male with medial medullary infarction accompanied by cerebral watershed infarction.

Findings: Brain digital subtraction angiography (DSA) showed mild stenosis in the basilar artery (BA) (Fig. 2A), plaque formation in the right internal carotid artery (ICA) without obvious stenosis (Fig. 2B), mild stenosis in the right middle cerebral artery (MCA) (Fig. 2C), and normal right vertebral artery (VA) (Fig. 2D), which appeared more dominant than the left in the extracranial portion (Fig. 2E). The left VA was occluded at the distal intracranial portion (Fig. 2F).

TECHNIQUE: DSA projections following vertebral (4ml/s Omnipaque 240 contrast), left SCA (8ml/s Omnipaque 240 contrast), right SCA (8ml/s Omnipaque 240 contrast), and right ICA injections (6ml/s Omnipaque 240 contrast).

Figure 2-2 (magnification of figure 2-1) 

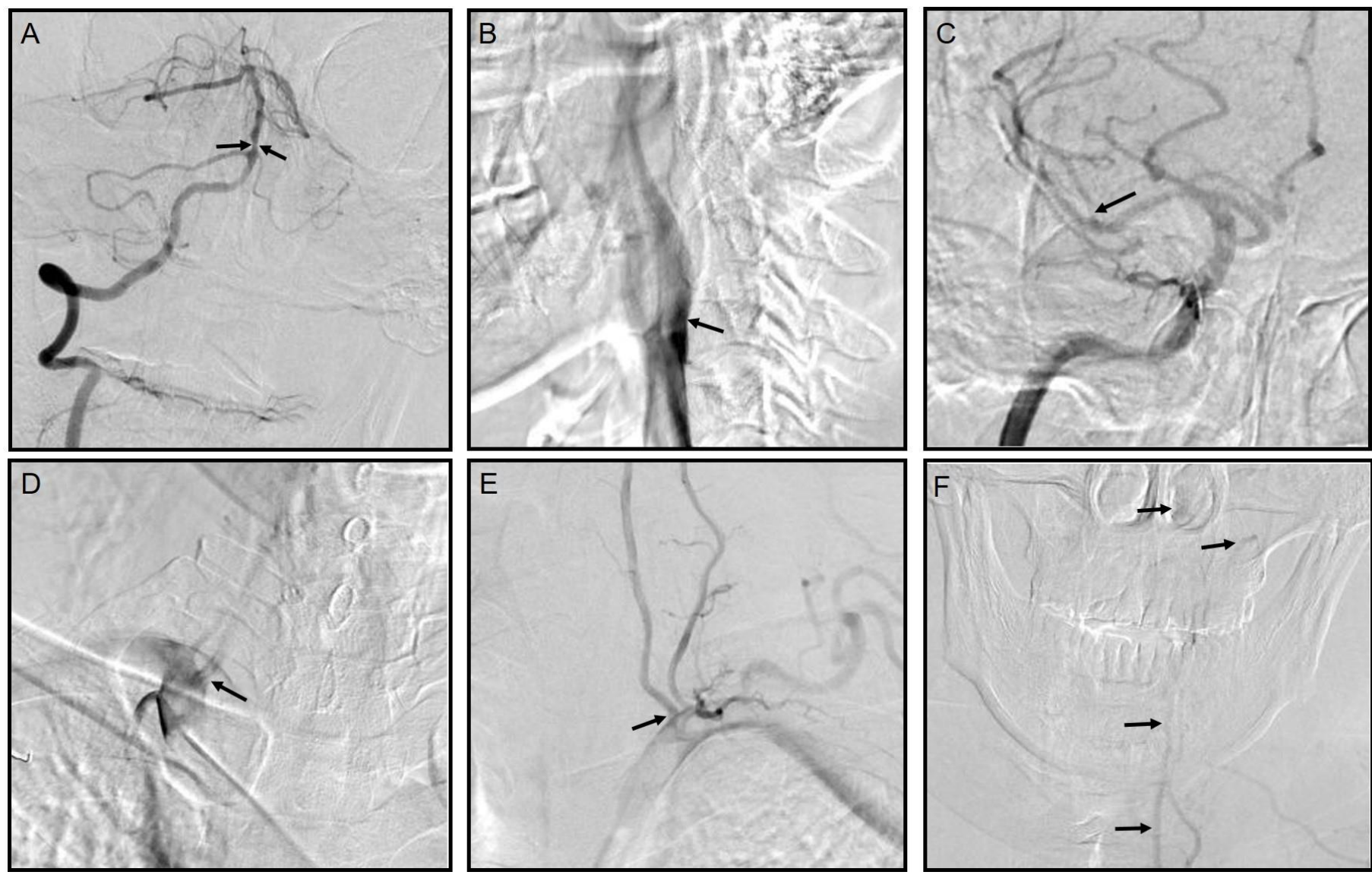

Figure 3: 50-year-old male with medial medullary infarction accompanied by cerebral watershed infarction. (magnification of figure 2)

Findings: Brain digital subtraction angiography (DSA) showed mild stenosis in the basilar artery (BA) (Fig. 2A), plaque formation in the right internal carotid artery (ICA) without obvious stenosis (Fig. 2B), mild stenosis in the right middle cerebral artery (MCA) (Fig. 2C), and normal right vertebral artery (VA) (Fig. 2D), which appeared more dominant than the left in the extracranial portion (Fig. 2E). The left VA was occluded at the distal intracranial portion (Fig. 2F).

TECHNIQUE: DSA projections following vertebral (4ml/s Omnipaque 240 contrast), left SCA (8ml/s Omnipaque $240 \mathrm{contrast})$, right SCA (8ml/s Omnipaque 240 contrast), and right ICA injections (6ml/s Omnipaque 240 contrast).

\begin{tabular}{|l|l|}
\hline Etiology & $\begin{array}{l}\text { The etiological classifications of infarctions are large-artery atherosclerosis (LA), small-vessel disease } \\
\text { (SV), arterial dissection (DI), cardiac embolism (CE), and stroke of un- determined etiology. }\end{array}$ \\
\hline Incidence & $\begin{array}{l}\text { Medial medullary infarction (MMI) is a rare stroke subtype, accounting for only 0.5-1.5\% of all strokes } \\
\text { [1.2], and bilateral MMI is even rarer [3]. }\end{array}$ \\
\hline Risk factors & $\begin{array}{l}\text { Atrial fibrillation, hypertension, smoking habits, diabetes mellitus, ischemic heart disease, and } \\
\text { dyslipidemia }\end{array}$ \\
\hline Treatment & $\begin{array}{l}\text { The same as cerebral infarction treatment, and to perform endotracheal intubation when respiratory } \\
\text { distress occurs. }\end{array}$ \\
\hline Symptoms & They include quadriplegia, dysarthria, hypoglossal palsy, respiratory failure, and so on. \\
\hline prognosis & The clinical outcome of bilateral MMI is usually poor [6]. \\
\hline Imaging findings & $\begin{array}{l}\text { Brain magnetic resonance imaging (MRI) shows a hyperintense "heart appearance" signal in the bilateral } \\
\text { anteromedial medullae. }\end{array}$ \\
\hline
\end{tabular}

Table 1: Summary table for Bilateral Medial Medullary Infarction 


\begin{tabular}{|l|l|l|l|l|}
\hline Diagnosis & X-Ray & CT & MRI & Key point \\
\hline $\begin{array}{l}\text { Bilateral Medial } \\
\text { Infurction }\end{array}$ & $\begin{array}{l}\text { Not } \\
\text { mentioned }\end{array}$ & $\begin{array}{l}\text { Not } \\
\text { mentioned }\end{array}$ & $\begin{array}{l}\text { MRI shows the classical } \\
\text { "heart" or "V/Y', appearance } \\
\text { at the ventral medulla in patients } \\
\text { with bilateral MMI [7, 8] }\end{array}$ & $\begin{array}{l}\text { A key point to differentiate between } \\
\text { syndromes is the evolution of the } \\
\text { clinical presentation; BMMI has a } \\
\text { more acute presentation [8]. }\end{array}$ \\
\hline $\begin{array}{l}\text { Guillain-Barre } \\
\text { syndrome }\end{array}$ & $\begin{array}{l}\text { Not } \\
\text { mentioned }\end{array}$ & $\begin{array}{l}\text { Not } \\
\text { mentioned }\end{array}$ & No abnormalities found. & $\begin{array}{l}\text { GBS has subacute evolution of } \\
\text { clinical presentation [8]. }\end{array}$ \\
\hline
\end{tabular}

Table 2: Differential diagnosis table for Bilateral Medial Medullary Infarction

\section{ABBREVIATIONS}

$\mathrm{ADC}=$ Apparent diffusion coefficient

ASA $=$ Anterior spinal artery

AWI $=$ Anterior watershed infarction

$\mathrm{BA}=$ Basilar artery

$\mathrm{C}$-IWI = Confluent internal watershed infarction

CWI $=$ Cortical watershed infarction

DSA $=$ Digital subtraction angiography

DWI $=$ Diffusion-weighted imaging

HDI = Hemodynamic impairment

ICA = Internal carotid artery

IWI $=$ Internal watershed infarction

MCA = Middle cerebral artery

MMI = Medial medullary infarction

MRI = Magnetic resonance imaging

P-IWI = Partial internal watershed infarction

PWI = Posterior watershed infarction

SCA = Subclavian artery

$\mathrm{VA}=$ Vertebral artery

$\mathrm{WSI}=$ Watershed infarction

\section{KEYWORDS}

Bilateral medial medullary infarction; Cerebral watershed infarction; Hemodynamic impairment; Diffusion-weighted imaging; Digital subtraction angiography

\section{Online access}

This publication is online available at:

www.radiologycases.com/index.php/radiologycases/article/view/3905

\section{Peer discussion}

Discuss this manuscript in our protected discussion forum at: www.radiolopolis.com/forums/JRCR

\section{Interactivity}

This publication is available as an interactive article with scroll, window/level, magnify and more features. Available online at www.RadiologyCases.com

\section{Published by EduRad}

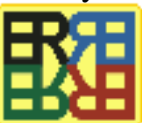

www.EduRad.org

\section{ACKNOWLEDGEMENTS}

We thank the patient and his family for their consent to publish the case report. We also thank Kunjun Wu who helped us during writing of this manuscript. 